\title{
e-Migrinter
}

$6 \mid 2010$

Rroms \& Gens du Voyage

\section{Les réfugiés congolais au Gabon : modes de circulation et d'installation dans un espace frontalier}

Thèse soutenue par Christian Wali Wali le 25 juin 2010 à l'Université de Poitiers

\section{Christian Wali Wali}

\section{OpenEdition}

\section{Journals}

Édition électronique

URL : https://journals.openedition.org/e-migrinter/1337

DOI : 10.4000/e-migrinter.1337

ISSN : 1961-9685

\section{Éditeur}

UMR 7301 - Migrinter

Édition imprimée

Date de publication : 15 octobre 2010

Pagination : 94-97

ISSN : 1961-9685

\section{Référence électronique}

Christian Wali Wali, «Les réfugiés congolais au Gabon : modes de circulation et d'installation dans un espace frontalier », e-Migrinter [En ligne], 6 | 2010, mis en ligne le 22 août 2019, consulté le 20 mai 2021. URL : http://journals.openedition.org/e-migrinter/1337 ; DOI : https://doi.org/10.4000/emigrinter.1337 


\section{Les réfugiés congolais au Gabon : Modes de circulation et d'installation dans un espace frontalier}

\section{Thèse soutenue par Christian Wali Wali le 25 juin 2010 à l'Université de Poitiers}

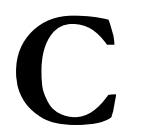

ette thèse traite la problématique générale de mobilités transfrontalières des réfugiés. L'analyse proposée montre que le Gabon a connu deux périodes importantes de flux des réfugiés. $\mathrm{La}$ première est celle de l'arrivée des enfants biafrais du Nigeria en 1968 et la seconde est celle des réfugiés congolais. En termes de gestion, de conditions d'accueil et du statut de réfugié à partir du droit international, ces situations ne sont pas identiques, et il y a une véritable spécificité du cas des réfugiés congolais due à la proximité frontalière, ethnique et politique.

Cette thèse a donc pour objet l'étude de la présence des réfugiés congolais au Gabon installés depuis la fin de la décennie 1990 et venus à la suite des différentes guerres civiles qui ont eu lieu au Congo. Le Congo a en effet connu durant cette décennie un climat politique instable provoqué par les leaders de la mouvance présidentielle et des partis de l'opposition qui se sont affrontés par milices interposées. Les antagonismes observés au cours de cette période sont des revanches entre acteurs politiques ayant animé le parti unique (19691990). Deux temps forts coïncidant avec deux moments électoraux marquent les instants de basculement de la confrontation politique au passage à l'utilisation des armes.

Le premier acte est la guerre de 19931994 qui résulte d'une transition mal négociée du passage de témoin entre Denis Sassou-Nguesso et Pascal Lissouba à la tête du pays à l'issue de l'élection présidentielle de 1992. Par le jeu de la construction et de la déconstruction des alliances politiques entre les différents acteurs politiques, le Congo bascule dans le conflit armé. Ayant bénéficié du soutien d'une coalition au sein de laquelle se trouvait le PCT (Parti Congolais du Travail) de Denis Sassou-Nguesso pour battre Bernard Kolélas à l'élection présidentielle, Pascal Lissouba ne respecte pas l'accord de gouvernement. Les élections législatives qui suivent donnent l'occasion à l'opposition de crier à la fraude et d'appeler à une désobéissance civile à laquelle le gouvernement répond par la violence.

Le deuxième acte de la crise politique congolaise se déroule à partir du 5 juin 1997 avec l'attaque de la résidence de Denis Sassou-Nguesso par des blindés de l'armée. Après un long séjour à l'étranger, ce dernier revient en janvier de cette année au pays. Mais au cours de ce séjour, Pascal Lissouba a réussi à faire de Bernard Kolélas son allié. A l'approche de l'élection présidentielle de 1997, les discours se durcissent et les conditions posées par les uns et les autres pour l'organisation de cette élection divergent. Un jeu d'accusations et de subterfuges politiques, retardant l'organisation des élections, s'engage alors. L'attaque de la résidence de Denis SassouNguesso entraîne la réaction immédiate de sa sécurité personnelle et de sa milice, les Cobras. Les pourparlers qui ont lieu à Libreville le 16 juin n'aboutissent à aucun compromis. L'échec de ces négociations conforte la dynamique des opérations militaires qui s'intensifient à l'horizon du 31 août qui est la date d'arrivée à échéance du mandat de Pascal Lissouba. Dès lors, des cohortes de populations partent du Congo pour se retrouver dans les pays environnants dont le Gabon. 
Parvenus au Gabon, ces populations congolaises vont s'installer particulièrement dans les provinces de la Ngounié, de la Nyanga, du Haut-Ogooué et de l'Estuaire. Les réfugiés congolais constituent le plus gros contingent et représentent aussi le plus important flux de réfugiés que le Gabon n'ait jamais reçu.

Au-delà de cette surreprésentation des réfugiés congolais par rapport aux autres nationalités, cette présence des réfugiés congolais est particulière dans la mesure où le Gabon partage avec le Congo la plus longue frontière de ce pays, que les deux pays ont été dans une même entité administrative (l'Afrique Equatoriale Française, AEF) pendant la colonisation et ont des populations frontalières de mêmes groupes ethniques. Cette situation est particulière quant à la gestion des réfugiés puisque par leur surreprésentation ils sont visibles, même si par leur appartenance aux mêmes groupes ethniques cette visibilité se dilue.

Ainsi, la question centrale qui a guidé cette recherche est celle de savoir comment l'asile se manifeste dans la zone frontalière gabono-congolaise pour les réfugiés congolais.

L'article 4 de la Convention de l'OUA (Organisation de l'Unité Africaine) relative à la gestion des réfugiés stipule que le statut de réfugié implique qu'il ne leur est pas permis de traverser la frontière, sous peine de le perdre. Malgré cela, on observe que les réfugiés congolais circulent et adoptent des stratégies de survie transnationales, ou tout le moins transfrontalières. Cela pose la question du statut de réfugié, de la souveraineté des États, de la citoyenneté des personnes en mouvement et du rôle des frontières, en ce sens qu'ils appartiennent à de multiples communautés (nationales, ethniques, etc.) et remettent en cause de ce fait la primauté de l'État comme le souligne Jennifer Hyndmann (2000).
L'hypothèse qui a guidé cette recherche sur le terrain était que les allers et retours des réfugiés congolais pouvaient s'inscrire dans le cadre de stratégies générales développées par les réfugiés pour une meilleure insertion dans leur commune d'accueil. Pour vérifier cette hypothèse, cette étude a consisté alors à comprendre les mécanismes d'insertion des réfugiés congolais à Lébamba en prenant en compte l'influence frontalière.

Les matériaux utilisés dans ce travail sont d'abord issus des différentes observations de terrain (de Lébamba au Niari), des récits de vie recueillis auprès des réfugiés congolais (3 à Lébamba et 5 à Libreville) et des formulaires remplis par les réfugiés (30) et les populations locales (87). Ces données sont complétées par les statistiques obtenues à la représentation régionale du HCR à Libreville et sur le site de cette institution, www.unhcr.org, mais aussi celles de la mairie de Lébamba et des différentes administrations gabonaises. Des entretiens aussi ont été faits avec les chercheurs, les autorités politicoadministratives et les habitants de Lébamba. Les entretiens et les questionnaires avec les réfugiés visaient à recueillir des informations sur les parcours des réfugiés depuis leur départ jusqu'à leur installation au Gabon mais aussi, sur leurs mobilités depuis leur arrivée pour reconstituer leurs principales étapes. Ceci a aidé à cerner la dimension de la circulation migratoire de ces personnes, des questions qu'elle soulève et la place que tient cette mobilité dans leur insertion locale. Il s'agissait de comprendre également les différents mécanismes utilisés par ces réfugiés pour leur insertion dans leur espace d'accueil. Par ailleurs, ce questionnement a permis d'analyser les politiques publiques en leur faveur et les effets de leur présence dans la commune de Lébamba.

Pour les populations locales interrogées, il s'est agi de recueillir leur perception de la présence des réfugiés congolais dans leur commune. Ainsi, même 
si les réfugiés sont bien acceptés par les populations, leur présence ne manque pas de soulever quelques interrogations. Leur exclusion dans certains domaines d'activités économiques (exploitation du vin de palme) et les noms par lesquels ils sont désignés («herbe Sassou») permettent de lire les limites de leur acceptation par les populations locales à Lébamba.

Les analyses conduites permettent de montrer que la localisation géographique des ethnies des deux côtés de la frontière et le voisinage de la ville frontalière refuge de Lébamba engendrent la mise en place de stratégies d'insertion particulière par les réfugiés et même par les populations générales qui vivent dans ces espaces frontaliers. La réalité de la frontière africaine, héritage de la colonisation, n'ayant pas détruit les liens inter-ethniques précoloniaux fait que contre l'État-nation, les populations transfrontalières et donc les réfugiés installés dans les zones frontalières imposent leurs règles de vie en contournant les lois étatiques. Les postes frontières sont de ce fait perçus parfois par ces populations comme des espaces de privation de liberté. L'étude des réfugiés congolais installés au Gabon en général, et à Lébamba en particulier montre, à travers la circulation transfrontalière, que les frontières africaines ne sont pas seulement des lignes de séparation et de discontinuité administrative, mais sont aussi des espaces à vocation de continuité culturelle, de réunification des peuples qu'elles avaient divisés et de redynamisation de ces zones grâce aux différents échanges à travers les activités commerciales notamment.

Ces activités commerciales aident également à comprendre que l'utilisation par les réfugiés congolais des deux territoires limités par la frontière étatique montre que la fuite, à l'origine contrainte, se transforme en une sorte de migration économique. Cette situation peut être cernée également à travers les déplacements des réfugiés congolais entre les villes gabonaises dans la mesure où on constate qu'environ 8 ans après leur arrivée au Gabon, les villes de premières arrivées dans lesquelles ils sont restés sont les villes ayant une activité économique développée (Moanda, Franceville, Mouila, Lébamba) et qu'on les retrouve aussi dans les villes non frontalières au Congo mais dans lesquelles l'activité économique est très développée (Libreville, Port-Gentil, Gamba).

Les allers et retours qu'effectuent les réfugiés congolais leur permettent également de mieux «accepter» leur situation de refuge. Le mouvement transfrontalier des réfugiés, tout en remettant en cause la frontière nationale, leur permet de mieux s'insérer dans une localité étrangère. C'est un moyen de mieux s'ancrer dans la durée sur leur lieu d'accueil. Cette volonté rencontre la volonté des autorités municipales qui souhaitent faire de Lébamba «une zone pilote pour l'intégration locale des réfugiés ».

A la frontière s'ajoute la dimension ethnique qui fait que les réfugiés et plus globalement les populations ont encore des difficultés à établir une distinction entre leur appartenance citoyenne et celle ethnique. Les perceptions contrastées des réfugiés congolais par les autorités et par les populations l'illustrent. Pour les autorités, il existe des réfugiés sur le territoire gabonais alors que pour les populations, il existe tout simplement des Congolais ou des Nzèbis. Les enquêtes de terrain permettent de voir que l'identité de réfugiés ou de Congolais est parfois récusée par certains habitants de Lébamba pour qui ces personnes ne sont que des Nzèbis.

Cependant, les analyses conduites dans cette thèse montrent que l'accueil des réfugiés congolais a mis en lumière la complexité des rapports politico-ethniques et familiaux des dirigeants politiques gabonais et congolais. En effet, au sommet de l'État gabonais, deux camps se sont opposés au sujet de la guerre de 1997 au Congo. Il y a le camp pro-Sassou représenté par sa fille Edith Lucie Bongo, dont 
l'influence a certainement été centrale dans l'aide de l'État gabonais à Denis SassouNguesso comme le note Patrice Yengo (2006). L'autre camp est représenté par Zacharie Myboto, membre influent du gouvernement gabonais, proche d'Omar Bongo, président du Gabon et époux d'Edith Lucie Bongo, d'ethnie nzèbi à laquelle appartient aussi Pascal Lissouba, l'autre protagoniste de la guerre congolaise avec qui il aurait des liens de parenté. Par ailleurs, Zacharie Myboto est le père de Chantal Myboto, qui a eu une longue liaison avec Omar Bongo et qui était sa conseillère dans les années 1990. Les affaires d'Etat entre le Gabon et le Congo sont difficilement compréhensibles sans prendre en compte les relations familiales et ethniques qui y interfèrent.

Christian Wali Wali

Docteur en Géographie MIGRINTER - UMR 6588 CNRS / Université de Poitiers c.waliwali@,voila.fr 